\title{
Subsidios de Educación: IMPACTO En la Migración Y CONVERGENCIA REGIONAL*
}

\author{
GonZALo DuRÁN**
}

During the last couple of decades, migratory movements in Chile have been minimal. Regions are far from achieving equality in per capita income, and migration has not been an equalizing force. Hence, there is marked persistence in regional inequity. This paper attempts to explain this lack of convergence. The main hypothesis is that there are some public policies that have an inhibiting effect over migration. In particular, the educational subsidies would act as an anchoring element. Using an enlarged version of the Harris and Todaro Model (1970) we conclude that in effect the "in school" food subsidy given by the Ministry of Education (for over 40 years) a powerful deterrent of migration between regions in Chile.

\section{JEL: $\mathrm{H} 52, \mathrm{O} 15, \mathrm{R} 23$}

Keywords: Migration, Convergence, Education Subsidies

\section{INTRODUCCIÓN}

Durante las últimas décadas los movimientos migratorios al interior de Chile han sido pequeños. Las regiones están lejos de igualar sus productos per cápita, situación que le daría persistencia a la inequidad regional y a la concentración de personas que habitan en la capital nacional.

Se calcula una velocidad de convergencia del 1,3\%, según lo cual se necesitarían 52 años para cerrar la mitad de la brecha entre una región pobre y una región rica, cifra que denotaría una no convergencia o un proceso de divergencia regional.

\footnotetext{
*Agradezco los comentarios de los profesores Raimundo Soto, Rodrigo Vergara, Luis Felipe Lagos, Fernando Ossa, Juan Pablo Montero y de un árbitro anónimo. Cualquier error u omisión es de mi responsabilidad.

** E-mails: giduran@puc.cl
} 
¿Qué es lo que genera esta aparente inmovilidad? ¿Cómo influye la migración en el proceso de convergencia regional? ¿Cómo una política -en particular la educación- puede inducir o inhibir migración?

La hipótesis es que la falta de convergencia regional en Chile se puede deber a los bajos niveles de movilidad, que serían producto de algunas políticas públicas, las cuales, sin necesidad de ser incorrectas, tienen un efecto indirecto sobre la migración. En este caso, se trabaja con subsidios a la educación. El argumento detrás de esta hipótesis es que la asignación de recursos fiscales dirigidos al establecimiento educacional, al correlacionarse con más apoyo para el alumno y con mejores resultados en cuanto a la calidad de la educación, inmovilizaría a la persona que está decidiendo migrar. A su vez, la falta de movilidad o poca migración es uno de los factores generatrices de la baja convergencia regional.

La metodología usada para abordar la pregunta es un modelo de regresión lineal, realizando estimaciones de corte transversal. Se trabaja con dos cortes en el tiempo que son: 1987-1992 y 1997-2002; esto, para cruzar la información correspondiente a los Censos de Población y Vivienda.

El artículo se organiza de la siguiente manera: en la sección 2 se presenta una revisión de la literatura, en la sección 3 el marco teórico, en la sección 4 se presentan los resultados empíricos y la sección 5 concluye.

\section{REVISIÓN DE LA LitERATURA}

\subsection{Revisión de la Literatura de Convergencia Regional}

Existe abundante investigación internacional sobre el tema. La tendencia de la mayoría es analizar los tipos de convergencia del modelo neoclásico. Una avenida es la de Barro y Sala-i-Martin (1992a), quienes desarrollan las pruebas empíricas de los modelos neoclásicos ${ }^{1}$ de crecimiento. Estas son Convergencia Beta y Convergencia Sigma. Según la Convergencia Beta: "Existe una covarianza negativa entre el crecimiento del producto o ingreso per cápita y el nivel inicial de dicho producto o ingreso". 2

Formalmente:

$$
\frac{1}{T}=\log \left[\frac{y_{T}}{y_{0}}\right]=x+\frac{\left(1-e^{-\beta T}\right)}{T} \log \left[\frac{y^{S S}}{y_{0}}\right]
$$

\footnotetext{
${ }^{1}$ Los modelos neoclásicos trabajan con funciones de producción que tienen productividad marginal positiva y decreciente, existen retornos constantes a escala y se cumplen las condiciones de Inada. Otra avenida es el enfoque de series de tiempo explotado por Bernard y Durlauf (1995). (Ver Apéndice 1).

${ }^{2}$ Es lo que se conoce como "Catch Up": las economías pobres crecen más rápido que las ricas y en el largo plazo convergen.
} 
donde $y$ corresponde al producto per cápita de cada región, con 0 periodo inicial y $T$ periodo final, $y^{S S}$ corresponde al valor del producto per cápita en estado estacionario. La Convergencia Beta necesariamente requiere que $\hat{\beta}$ sea mayor a 0 . Barro y Sala-i-Martin (1991) llegan ${ }^{3}$ a que para el Reino Unido (11 regiones) el beta es de $3,37 \%$, para Alemania (11 regiones) es de 2,3\%, en tanto que para los Países Bajos es de 4,96\%. Al comparar los estados de Estados Unidos (48 estados) con las prefecturas de Japón (47 prefecturas), tomando datos desde 1880-1988, Barro y Sala-i-Martin (1992b) llegan que el beta es de 2,5\% para el primer caso y de 3,0\% para el segundo. Para Chile, Fuentes (1996) concluye 4 que la mitad de la brecha entre región pobre y región rica se cerraría en 52 años, esto es, con un beta estimado de 1,32\%. Díaz (2002) y Díaz y Meller (2003) arriban a similares conclusiones con velocidades de convergencia que fluctúan entre $1,1 \%$ y 2,1\%. Oyarzún y Araya (2001) ocupando el enfoque de series de tiempo de Bernard y Durlauf(1995) concluyen que existe una gran resistencia a la convergencia y demuestra la existencia de regiones "puzzles", 5 que no logran converger en el largo plazo.

Convergencia Sigma: "Se aprecia una disminución en el tiempo de la dispersión del producto o ingreso per cápita de las distintas regiones en análisis". Formalmente:

$$
\sigma_{t}^{2}=\left(e^{-2 \beta}\right) \sigma_{t-1}^{2}+\sigma_{u}^{2}
$$

donde sigma es la desviación estándar del logaritmo del producto (o ingreso) per cápita de cada región. En este caso se espera que la varianza decrezca a través del tiempo. Díaz et al. (2002) concluyen de manera contundente que la dispersión se ha mantenido constante en el tiempo proporcionando así evidencia de la persistencia temporal de las disparidades del producto per cápita a nivel de regiones. ${ }^{6}$

Como conclusión de esta línea investigativa existe consenso de que la velocidad de convergencia es relativamente lenta, lo cual implica que deberán pasar muchos años para que los niveles de producto (o ingreso) per cápita entre regiones se igualen. ${ }^{7}$

Una posible explicación que han dado algunos economistas se centra en el rol de la migración ${ }^{8}$ sobre la convergencia. Barro y Sala-i-Martin (1991) argumentan que tiene un impacto positivo sobre la velocidad de convergencia, esto,

${ }^{3}$ El período va desde 1950 hasta 1985 .

${ }^{4}$ En el caso de convergencia no condicionada (clásico). Al condicionar por variables ad hoc aumenta la velocidad.

${ }^{5}$ Es el caso de las regiones I, II y XII, que no logran cointegrar y, por lo tanto, no convergerían.

${ }^{6}$ Veáse también Riffo (1999), quien al respecto realiza un análisis de largo plazo.

${ }^{7}$ Según los cálculos de Díaz (2002), para alcanzar un PIB/Cápita de \$2.000.000 (\$ de 1986), la II Región lo haría en el 2008, la III en el 2020, en tanto que la VII en el 2050, la XI en el 2060 y la IX en el 2068, ello según crecimiento 1975-1998.

${ }^{8}$ Díaz (2002) sugiere que la movilidad puede favorecer convergencia, a través de las personas que dejan una región que ofrece bajo salario para irse a otra región que ofrece mayor salario. De tal manera que mientras mayor sea la diferencia de salarios, mayor será la incitación a migrar. 
mediante el ajuste en el mercado laboral, en ello, el nivel de capital humano que posean los migrantes es importante; para el caso de Estados Unidos (48 estados) el rol de la migración es importante en la velocidad de convergencia y la aumentaría en $1 / 3$ cuando se incorporan las tasas de migración neta en la regresión de la Convergencia Beta; sin embargo, advierten que el impacto es bastante menor cuando se pone atención en el nivel de capital humano de los migrantes.

\subsection{Revisión de la Literatura de Migración}

La literatura de migración ha sido construida mayoritariamente por sociólogos, demógrafos, geógrafos y economistas. Esta sección apunta a una revisión de la contribución de estos últimos. Larry Sjaastad en un trabajo pionero señalaba: “...tratamos la migración como una inversión, que aumenta la productividad de los recursos humanos, una inversión que tiene costos y que también rinde beneficios..." [Sjaastad (1962), pp. 83]. Se identifican determinantes monetarios y no monetarios presentes en el proceso migratorio. Entre los determinantes monetarios la contribución de Todaro (1969) y Harris y Todaro (1970) marcan un hito al desarrollar un modelo sencillo de equilibrio general con dos sectores, ${ }^{9}$ concluyendo de manera contundente lo que en palabras era simple: las personas mediante una decisión que maximiza su bienestar, eligen aquel sector que remunere mejor su productividad marginal. El diferencial de los salarios entre sector urbano y un sector rural era uno de los determinantes más potentes.

En Mincer (1978), se desarrolla un modelo de decisiones públicas, descontextualizando de este modo la individualidad que tendría el modelo de Harris y Todaro (1970). Analiza el caso de decisiones familiares en el momento de migrar y para eso el autor desarrolla un modelo matrimonial (o de migración atada), evaluando distintos escenarios posibles en cuanto a la situación laboral y civil del esposo y la esposa. Concluye que la migración tiende a ser mayor en personas divorciadas, separadas, solteras y en matrimonios sin hijos. Asimismo, concluye que la migración tendería a reducir el desempleo del hombre, pero aumenta el desempleo de la mujer, y una de las causas sería el proceso de ajuste asociado en la inserción de los hijos a la nueva región.

En Chile, destaca la contribución de Aroca y Hewings (2002), quienes desarrollan un modelo tradicional de migración a la Harris Todaro, estableciendo un grupo de variables que serían las determinantes en la decisión al momento de moverse de región. Los resultados empíricos demuestran que las diferenciales de salarios entre las regiones de Chile incentivan movimiento; se llega a que los principales centros urbanos de la época (RM, VIII, V) tienen los efectos de atracción mayor.

Fuentes (1996) analiza el impacto de la migración en la convergencia regional para Chile concluyendo que al incorporar migración, aumenta la velocidad de convergencia. En particular, para la regresión de convergencia no condicionada

\footnotetext{
${ }^{9}$ Bhattacharya (1994) y Bhattacharya (1998) expanden el análisis con un tercer sector.
} 
por otras variables, el beta estimado aumenta de $1,32 \%$ a $1,77 \%$, lo que reduce el tiempo ${ }^{10}$ en aproximadamente 10 años. A similares conclusiones llegaron Barro y Sala-i-Martin ${ }^{11}$ (1991) y (1992b). Esto es, la migración sí tiene efecto sobre la velocidad de convergencia del modelo neoclásico.

\subsection{Revisión de la Literatura de Educación}

La literatura económica relacionada con educación generalmente muestra la importancia que tiene la adquisición de capital humano como semilla y posterior motor en el desarrollo de las personas y del país. Se trata del clásico argumento minceriano, ${ }^{12}$ según el cual el retorno monetario de la educación está en directa proporción a los años de educación recibida. Sin embargo, en Becker (1993), Mincer es mencionado a modo de referencia, dejando de lado la identidad contable y demostrando de paso que el tema del capital humano es algo integral, y que tiene muchas aristas que no son tomadas en cuenta. Becker menciona que tanto salud física como psíquica son un tipo de inversión en capital humano. De este modo, el hecho de que un país presente bajos niveles de hambruna incide en una menor tasa de mortalidad, la cual a su vez significa una mayor esperanza de vida al nacer y con ello aumenta el valor presente de los ingresos que se obtienen, producto de la inversión en capital humano. De esta vertiente de la literatura se desprende que las posibilidades de movilidad social estarán fuertemente determinadas por el nivel de "educación integral". Es por esta razón que los individuos destinan importantes cantidades de recursos al ítem educativo y es también deber del Estado destinar recursos a la educación, para así cubrir los sectores más desposeídos. Estos recursos se distribuyen en diferentes programas, dando origen a los subsidios en todos los niveles educacionales. A través de los programas de ayuda, los estudiantes son financiados en algún aspecto del proceso educacional para así asegurar la estadía en el sistema.

Gallego (2002) enfatiza un rasgo central en la literatura empírica vinculada a la selección del establecimiento, mostrando que tanto los estudiantes como sus familias tienen elevados costos, si se cambian de establecimientos educacionales durante el periodo de vida escolar. Lo anterior hace más probable que los que eligen la escuela donde estudiar sean mayoritariamente individuos que han vivido algún fenómeno migratorio o que inician su vida escolar. En este punto conviene señalar la situación que experimentan aquellas personas que reciben asistencia del Estado a través de los subsidios de educación. González et al. (2002) advierten que la implementación de ciertos programas gubernamentales dirigidos a la

${ }^{10}$ Para calcular el número de años que tarda en igualarse la mitad de la brecha entre región pobre y región rica, se puede realizar de manera recursiva. Sin embargo una especificación que han dado algunos economistas [Higuera (2004)] es: Tiempo $=\ln 2 / \hat{\beta}$.

${ }^{11}$ En ambos trabajos, así como en la investigación de Fuentes (1996) las tasas de migración se incorporan al lado derecho en la fórmula de la convergencia beta. Ver Apéndice 2.

${ }^{12}$ En 1974, Jacob Mincer da a conocer su identidad contable, más conocida como la Ecuación de Mincer: $\operatorname{Ln} W=\beta_{0}+\beta_{1} E d+\beta_{2} E x+\beta_{3} E x q+\mu$, donde W: Salario, Ed: Años de Educación Recibida, Ex: Experiencia, Exq: Años de experiencia al cuadrado. 
educación provocan un conflicto entre excelencia y equidad. Se trata de un tradeoff entre más puntos SIMCE (Sistema de Medición de Calidad de la Educación) versus una mayor atención a personas que presentan carencias de aprendizaje. Acuñan el término de "recursos diferenciados" refiriéndose así a la focalización que existe en la distribución y entrega de los recursos monetarios asociados a educación que reciben las comunas, establecimientos educacionales y alumnos; los autores señalan que un sistema de focalización que entrega los recursos en proporción al porcentaje de alumnos en situación vulnerable tiene la limitación de la no portabilidad de estos recursos adicionales. Según lo cual, si un alumno se cambia de establecimiento perdería el apoyo. ${ }^{13}$

Por último, Sapelli y Aedo (2001) analizan el sistema de "vouchers" 14 a la educación, realizando el análisis desde la perspectiva de un subsidio a la demanda (subsidio por estudiante). El sistema de vouchers en Chile no permite obtener conclusiones relativas a migración, ya que el estado garantiza un voucher que es gratuito a toda persona que se quiera educar.

\section{MARCO TEÓRICO}

\subsection{Maximización de la Función de Utilidad}

Los estudios teóricos sobre migración se enmarcan en tópicos de bienestar. Se supone un agente que maximiza su función de utilidad. La migración se toma como el resultado de las variaciones entre la utilidad en diferentes ubicaciones espaciales. La reubicación (en la región de destino) se produce si la utilidad esperada del migrante es mayor que la utilidad de permanecer en la región actual. Los individuos, por su naturaleza heterogénea, tienen funciones de utilidad diferentes con lo cual se pueden encontrar diferencias en los beneficios netos de vivir en una ubicación específica.

Se asume un individuo $k$ que evalúa cambiarse desde la región $i$ hacia la región $j$. El retorno esperado para el individuo $k$ en el momento del tiempo $t$ se obtiene de un proceso de maximización de diferenciales de utilidad esperada:

$$
\left.E\left(R_{k t}\right)=\underset{(X, Z)}{\operatorname{Max}}\left[\begin{array}{c}
T \\
E \int e^{-r t} \\
0
\end{array} U_{k j}(X, Z)-U_{k i}(X, Z)\right\} d t-C M_{k i j}\right]
$$

donde $X$ es un conjunto de bienes y $Z$ otras características. Los costos de moverse desde la región $i$ hasta la región $j$ se denotan por $C M$. Con la precondición de que la ecuación (3):

\footnotetext{
${ }^{13}$ Otra limitación que se señala es que no promueve la competencia entre establecimientos y no genera empowerment (los apoderados no se sienten en posición de exigir buena educación).

${ }^{14}$ Es un cupón asociado al alumno, en la práctica son las Unidades de Subvención Escolar (USE). Veáse también Sapelli y Vial (2002), Sapelli (2002).
} 


$$
e^{-r t}\left\{U_{k j}(X, Z)-U_{k i}(X, Z)\right\} d t-C M_{k i j} \geq 0
$$

Resolviendo, el individuo $k$ se mueve desde $i$ hacia $j$ si la diferencia de utilidades marginales de cada variable optimizada es mayor a cero. Formalmente, de las condiciones necesarias de primer orden:

$$
\begin{aligned}
& u_{x}^{j_{j}} e^{-r t}-u_{x}^{i} e^{-r t} \geq 0 \quad \Rightarrow \Delta u_{x} \geq 0 \\
& u_{z}^{j} e^{-r t}-u_{z}^{i} e^{-r t} \geq 0 \quad \Rightarrow \Delta u_{z} \geq 0
\end{aligned}
$$

La ecuación (3) representa la condición fundamental de la conducta del migrante: "El individuo maximiza una función de utilidad de acuerdo a sus propias preferencias".

En los modelos de migración lo tradicional es que se trabaje a nivel personal, sin distinguir entre individuo y familia. En este caso, se sigue la línea de Mincer (1978) y se trabaja en el contexto familiar. Con sólo un miembro en la familia se tiene que $k=1=f$, desde el momento en que la familia tiene dos o más miembros y bajo el supuesto de que la decisión de migración es Pareto óptima se tendrá:

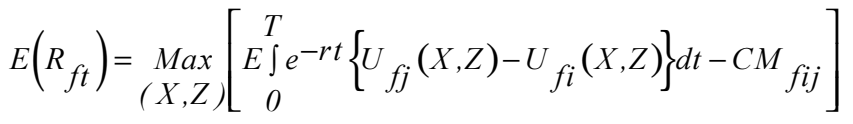

donde:

$$
R_{f t}=\sum_{k} R_{k} \quad U_{f}=\sum_{k} U_{k} \quad C M_{f}=\sum_{k} C M_{k}
$$

Jacob Mincer presume que las familias tenderían a ser menos móviles que lo que podría llegar a ser un individuo. Lo anterior se debería esencialmente al aumento en los costos de migrar cuando la familia tiene hijos, sobre todo en la etapa de formación escolar. 


\section{GRAFICO 1}

MIGRACION Y DIFERENCIAS SALARIALES

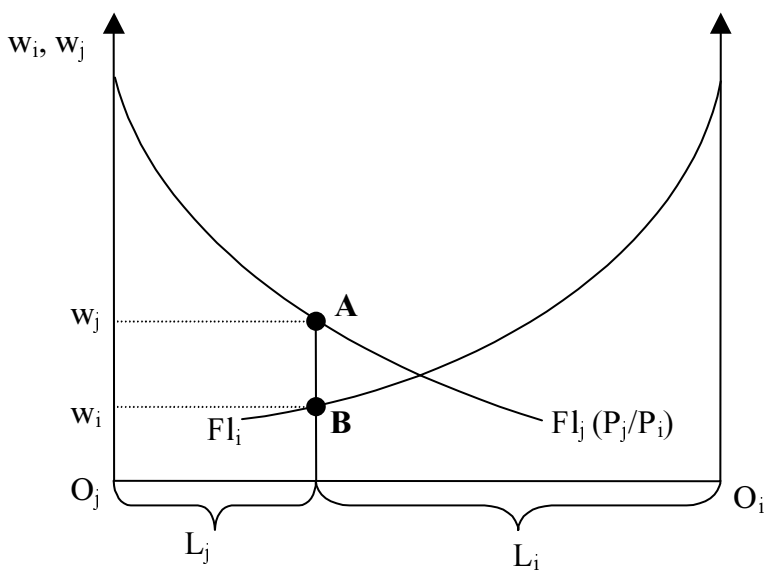

El modelo de Harris y Todaro (1970) ${ }^{15}$ es uno de los modelos pioneros en el área migratoria. Mediante una sociedad en la que existe un sector urbano y uno rural, se concluye que los movimientos migratorios se producen siempre y cuando el ingreso futuro esperado en el sector urbano supere el ingreso esperado futuro en el sector menos desarrollado. Sin embargo, la gran limitante del modelo HT radica en que el núcleo de la decisión migratoria está en el individuo, obviando las relaciones de interdependencia familiar. De todas maneras, la relevancia del modelo HT, no se discute y en Bhattacharya (1993) se comprueba que, afrontando la mencionada debilidad de HT, se ayudaría a explicar que la ausencia de movimientos migratorios ante niveles salariales distintos no implica, para nada, que el individuo decida irracionalmente, sino que tiene en cuenta otro tipo de dotaciones como, por ejemplo, bienes y servicios públicos, y subsidios a la educación.

En el Gráfico 1 se aprecia la distribución de la fuerza laboral (eje de las abscisas). En este caso, el sector $j$ tiene mayor remuneración al factor, por lo que la transición hacia el equilibrio indica que los individuos migran hacia el sector $j$, con lo cual aumenta $L_{j}$ y al mismo tiempo disminuye la productividad marginal del factor en dicho sector.

El modelo de HT explota los diferenciales en los salarios. Según esto, las personas se desplazan hacia donde la remuneración es mayor y la corriente migratoria conlleva a que la razón de uso $\mathrm{K} / \mathrm{L}$ disminuya en la región de destino. En el Gráfico 2, se ve (en el contexto neoclásico) cómo el aumento de la fuerza laboral vía inmigración hace caer el salario.

\footnotetext{
${ }^{15}$ De ahora en adelante HT. Otros modelos bisectoriales son Lewis (1954) y luego Ranis y Fei (1961).
} 
GRAFICO 2

MIGRACION Y MODELO NEOCLASICO

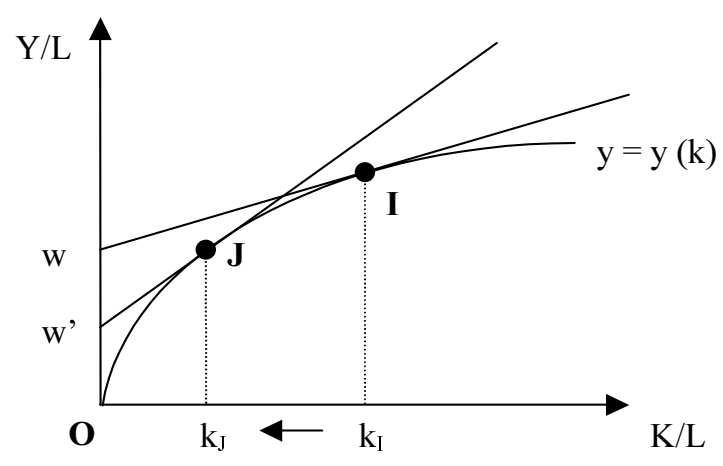

La migración se convierte en un mecanismo a través del cual se pueden resolver desequilibrios en el mercado laboral. ${ }^{16}$ La dinámica es como sigue: los trabajadores desempleados de aquella región económicamente deprimida pueden buscar trabajo migrando a una región que sea más próspera. Es así como el desempleado de la región i, encontraría su "match" trabajando en la región $j$. Básicamente, las regiones que tengan una tasa de desempleo mayor deberían tener los flujos emigratorios relativamente mayor (al menos en el corto plazo) y las regiones que tengan mayores puestos de trabajo tendrían los flujos inmigratorios mayores.

\subsection{Determinantes de la Migración}

¿De qué depende que la gente se mueva de un lado a otro? Resulta relativamente obvio que existen muchas razones de por qué las personas podrían moverse desde una región a otra. ${ }^{17}$ La teoría económica señala que las diferencias en salarios es un factor relevante para migrar, Hicks (1948) señalaba: “... las diferencias en las ventajas económicas netas, principalmente diferencias en salarios, son las causas principales de la migración" [Hicks (1948), pp. 76]. Trabajos previos, ${ }^{18}$ descubrieron que existían indicadores no monetarios que hacían moverse y es frecuente que en los modelos de migración siempre se controle por ellos.

Es común que las personas se sientan atraídas por grandes ciudades que ofrecen una mayor cantidad de bienes de consumo, mayor acceso a servicios

\footnotetext{
${ }^{16}$ Aroca y Hewings (2002).

${ }^{17}$ En Mincer (1978) se señala el caso de los matrimonios como uno de los determinantes al momento de moverse de región, reafirmando su punto de vista de que la migración es primero que todo una decisión no individual.

${ }^{18}$ Sjaastad (1962), Greenwood (1975), Mincer (1978).
} 
públicos, recreación, clínicas, colegios, tecnologías de la información, etc. Es lo que en la literatura de migración se conoce como el "Efecto Luces de la Ciudad" (Coeymans, 1982). Este aspecto fue pieza fundamental en el proceso migratorio rural-urbano.

Entre los determinantes más difíciles de medir y, por ende, poco reconocidos en investigaciones contemporáneas destaca el "Efecto Psíquico". En este punto retomamos la línea teórica de Jacob Mincer. Es decir, las decisiones de migrar son decisiones familiares y el hecho de cambiarse de región puede tener efectos psíquicos importantes, los que estarían representados en pérdidas de grupos de referencia, amigos, familiares, etc. Lo anterior es más fuerte en la etapa de formación escolar. En Greenwood (1975) se señala: “... los costos psíquicos se pueden convertir en un permanente costo de transporte por la necesidad de visitar el lugar de origen como una manera de negar la agonía de dejar a la familia y a los amigos..." (Greenwood, 1975, pp.404).

Un tercer determinante migratorio es el llamado "Efecto Distancia". La migración decrece sustancialmente a medida que aumenta la distancia entre una y otra región. Para el tratamiento empírico, es común que se suponga una medida del costo de transporte. En este sentido, la formación de "clusters", o regiones colindantes lideradas por un gran centro urbano, promueve la migración al interior del cluster, pero se dificulta entre clusters. ${ }^{19}$ Esta agrupación en clubes está estrechamente relacionada con las teorías de ubicación espacial, que, a su vez, se relacionan con los test de convergencia. El punto de vista de Higuera (2004) es que el espacio es un factor importante para explicar el comportamiento de la distribución del producto per cápita regional. Es decir, las regiones tienden a estar rodeadas de regiones con niveles de producto per cápita similares. La formación de clusters de regiones pobres y regiones ricas sería una consecuencia de la estructura espacial de cada territorio geográfico.

El cuarto determinante es el llamado "Efecto Información". Es más probable que exista migración hacia una ciudad sobre la cual se tiene relativo conocimiento. Este efecto se potencia con el "Efecto Luces". Las urbes entregan gran cantidad de información que hace disminuir la incertidumbre de la región $j$. También se relaciona con el "Efecto Distancia". A mayor distancia aumenta la incertidumbre y disminuye la información, lo cual potenciaría la formación de estructuras espaciales o clusters. El "Efecto Información" genera lo que en la literatura se conoce como Migración Especulativa y Migración Contratada. La primera ocurre como parte del proceso de búsqueda de trabajo, mientras que la segunda es una consecuencia de éste. La probabilidad de éxito en la migración especulativa aumenta en tanto aumenta la información sobre la región $j$.

\footnotetext{
${ }^{19}$ En el Anexo 3 se aprecia la distribución de Chile Regional; la idea de los clusters (o vecindades) es que en la migración hacia el centro urbano de la región $j$, la probabilidad de que el lugar de origen sea de provincias del cluster asociado a ese centro urbano es $>$ de que sea de provincias de clusters de otro centro.
} 
Otro determinante que se debe considerar es el nivel de capital humano, que llamaremos "Efecto Capital Humano". El nivel de escolaridad logrado por el individuo se relaciona positivamente con la migración. El punto de vista de Ritsilä y Ovaskainen (2001) es que un mayor nivel de capital humano abre un abanico de posibilidades de empleo más amplio que el que tendría una persona con nulo capital humano. En consecuencia, las personas más "escolarizadas" serán geográficamente más móviles. El supuesto detrás de eso es que si la persona $k$ no encuentra empleo en la región $i$ puede moverse hacia la región $j$ y tendrá mayor posibilidad de éxito, en cuyo caso el capital humano es una ventaja competitiva. Sin embargo, existe otra dimensión y es que la persona se va movilizar hacia aquella región que presente mayor número de años de escolaridad. ${ }^{20}$ Lo anterior es correcto cuando se trabaja con gobiernos descentralizados, en donde la decisión del número de años de estudios obligatorios se toma de manera autónoma en cada región (o en cada país). En el caso de estar insertos en un país manejado por un gobierno central esta segunda dimensión ya no es gravitante en la migración. ${ }^{21}$

El sexto y último determinante es lo que he denominado "Efecto Vaso de Leche". Los programas de ayuda social, con apoyo directo en la población más desposeída, serán llamados programas "vaso de leche" por la connotación de ser ayuda de primera necesidad. Un mal diseño de estos programas hará que la persona $k$ no tenga incentivo a moverse desde la región $i$ hacia la región $j$.

\section{GRAFICO 3 \\ EFECTO VASO DE LECHE}

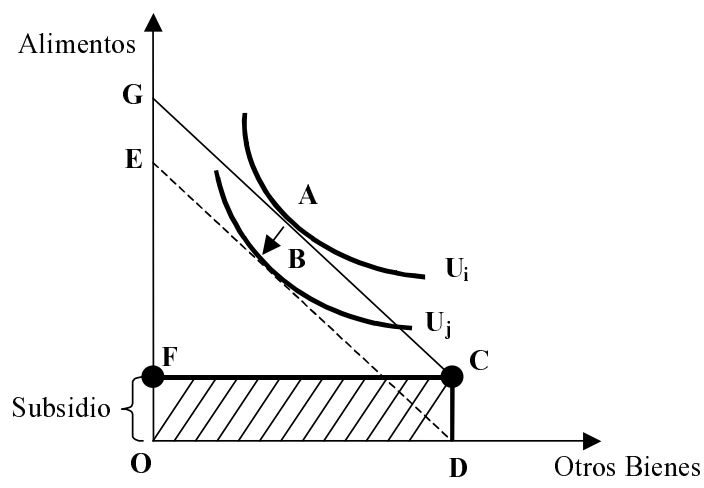

Este efecto se ilustra en el Gráfico 3. Inicialmente la persona $k$ se ubica sobre la línea de riqueza GC, en un punto como A, donde la curva de indiferencia es

\footnotetext{
${ }^{20}$ Una región que invierte más en educación y formación puede crecer más rápidamente que otra que invierte menos; a su vez estos niveles incidirán en los flujos migratorios explicando con ello una posible divergencia o no-convergencia.

${ }^{21}$ Sin embargo, como se verá en el análisis empírico, los niveles de escolaridad promedio entre regiones generalmente difieren y están muy debajo de los años obligatorios; por lo tanto, existe un elemento de deserción escolar a considerar.
} 
tangente. Sobre esta línea GC el individuo estando en la región $i$ recibe un subsidio -en este caso alimentación- equivalente al tramo OF. En este escenario, un movimiento desde la región $i$ hacia la región $j$ equivale a perder el mencionado subsidio, en cuyo caso, disminuye la riqueza del individuo en el monto GE, representado en unidades de alimento: nótese que los "Otros Bienes" han quedado inalterados, en un punto D. Ahora, en la región $j$, el individuo logra una utilidad $U_{j}$ que es menor a $U_{i}$. Esto es así, porque en el diseño de la asignación de ciertos subsidios existen mecanismos que no garantizan que un alumno al cambiarse de ubicación geográfica mantenga los mismos beneficios que tenía en un comienzo. En el caso de la alimentación, cambiarse de región puede provocar perder el vaso de leche.

\subsection{Sistema de Subvenciones a la Educación en Chile: Antecedentes Locales}

Por mandato constitucional la educación básica y media es obligatoria en Chile y por esta razón el Estado garantiza el acceso a través de subvenciones para el financiamiento de la educación. ${ }^{22}$

Más del $90 \%$ de la población escolar básica y media recibe educación en establecimientos financiados por aportes fiscales..$^{23}$ El ciclo de educación escolar comprende un periodo de 12 años mínimo y está definido para todos los chilenos hasta los 21 años de edad.

\subsubsection{Sistema Pagos Gestionados por la Coordinación Nacional de Subven- ciones $(\mathrm{CNS})^{24}$}

Son exactamente 20 los tipos de pagos ${ }^{25}$ que la CNS dispone para apoyar a la educación; los recursos fiscales son inyectados al sistema educativo según normativas preestablecidas, identificándose tres categorías de apoyo: al establecimiento, al director y a los alumnos. El apoyo al establecimiento y al director decanta de manera indirecta en el alumnado. El ánimo del apoyo sigue dos premisas básicas: mejor calidad de la educación e igualdad de oportunidades al momento de recibir educación.

Existen ciertos subsidios, que otorgando el beneficio a la persona en la región $i$, no lo otorga a esa misma persona en la región $j$. Es lo que he denominado

\footnotetext{
${ }^{22}$ Decreto Fuerza Ley No 2, de 1989, sobre Subvención del Estado a Establecimientos Educacionales, en Diario Oficial de la República de Chile, 9 de marzo 1993.

${ }^{23}$ En Chile existen tres tipos de establecimientos educacionales: Municipales que son financiados íntegramente por el Estado y administrados por los municipios, los Particulares Subvencionados que los administra el sector privado, pero también reciben financiamiento estatal, y los Particulares Pagados.

${ }^{24}$ En Chile, el sistema de Subvenciones está encargado al Ministerio de Educación, quien a través de la Coordinación Nacional de Subvenciones organiza y gestiona el apoyo a la educación.

${ }^{25}$ Mineduc (2004), "Descripción de Pagos Gestionados por la Coordinación Nacional de Subvenciones".
} 
el "Efecto Vaso de Leche". ${ }^{26}$ Es en este caso que una política de subvenciones, sin necesidad de ser incorrecta, tiene efectos indirectos sobre otros fenómenos económicos. En este grupo de subsidios entran: la "Subvención de Internado" y la "Subvención por Reforzamiento Educativo".

El primero de estos subsidios está dirigido a acoger a los alumnos que por condiciones geográficas no pueden acceder al sistema educativo, porque los costos de ir y volver hacia el establecimiento son muy elevados. La selección de quien recibe el subsidio se realiza mediante su condición de vulnerabilidad. ${ }^{27}$ De este modo, un alumno viviendo en la región $i$ puede tener subvención de internado -su condición de vulnerabilidad se lo garantiza-; sin embargo, ese mismo alumno en la región $j$ (que en este caso también necesita internado por su ubicación geográfica) puede que no tenga la subvención de internado debido a que su condición de vulnerabilidad ya no es la misma, ${ }^{28}$ aun cuando su condición económica si lo es. En este caso, la única opción de educarse será cubriendo los costos de transporte para llegar al establecimiento.

En el segundo caso, el alumno recibe reforzamiento docente ( "amenity" en la enseñanza, es gratis) en la región $i$, pero no necesariamente en la región $j$. La subvención la reciben sólo algunos establecimientos que son los que imparten los cursos de reforzamiento y, dentro de éstos, quienes reciben el apoyo son alumnos que por su condición de vulnerabilidad así lo requieren. El apoyo, además de los reforzamientos incluye -en algunos casos- alimentación proporcionada por la Junta Nacional de Auxilio Escolar y Becas.

\subsection{Otras Bases del Sistema de Apoyo Educacional.}

\subsubsection{Junta Nacional de Auxilio Escolar y Becas (JUNAEB)}

La JUNAEB es el ente encargado de la construcción del Indice de Vulnerabilidad (IVE), según el cual se focaliza la atención que ellos mismos proporcionan. ${ }^{29}$ El IVE se construye sobre la base de una encuesta que mide vulnerabili$\operatorname{dad}^{30}$ de alumnos que asisten a una escuela. La encuesta es enviada a las escuelas municipales y particulares subvencionadas de todo el país. El profesor jefe, en conjunto con el apoderado, llenan la encuesta, la cual se realiza en $1^{\mathrm{er}}$ año básico y en $1^{\text {er }}$ año medio. Aquella escuela que tiene más "alumnos con daños" es más

\footnotetext{
${ }^{26}$ Más adelante se aplica de manera literal (JUNAEB).

${ }^{27}$ Para más detalle ver la sección adyacente: Junta Nacional de Auxilio Escolar y Becas.

${ }^{28}$ De hecho, aún si la condición de vulnerabilidad fuera la misma, existe el problema de las vacantes en el internado.

${ }^{29}$ JUNAEB: Junta Nacional de Auxilio Escolar y Becas: su misión es entregar ayuda a los alumnos más vulnerables de Chile, ayuda que se materializa en alimentación (se ejecuta en el establecimiento educacional), salud estudiantil y apoyo integral. Institucionalmente depende del Ministerio de Educación.

${ }^{30} \mathrm{La}$ encuesta ha sido ampliamente validada.
} 
vulnerable y necesita más recursos. ${ }^{31}$ El IVE es el único indicador de pobreza en comunas rurales. La JUNAEB toma los datos y construye un indicador de daño para el establecimiento. Con eso se construye el IVE a través de un modelo estadístico. El Rango del IVE es de 0-100. Sobre 68, si se habla del Programa de Alimentación Escolar (PAE), ${ }^{32}$ se entregarían las raciones de mayores calorías (M1.000), entre 26- 68 está la mayoría (M700), entre 20-26 sólo desayuno (no son tan vulnerables). Sobre 50 se considera vulnerabilidad grave. Para ejemplificar, tomemos el caso de que el establecimiento obtenga un IVE 50, esto quiere decir que el $50 \%$ de los alumnos de la escuela son vulnerables. Si son 100 alumnos, 50 reciben ración, aquéllos serán los más vulnerables y dentro de cada establecimiento se tiene identificado al alumno que recibe y al que no recibe alimento, ${ }^{33}$ siendo el director y el profesor quienes las distribuyen. ${ }^{34}$ La hipótesis de esta investigación es que los alumnos no van a moverse de región, ya que nada les asegura que la escuela de destino sea igual de vulnerable, situación que le haría mantener el beneficio. De hecho, lo que más probable es que pierda el beneficio. El alumno, en el caso extremo, podría moverse desde la región $i$ de un establecimiento que tiene un IVE de $80 \%$ a la región $j$ a un establecimiento con un IVE de $20 \%$; en ese caso, el alumno pasa de un establecimiento donde recibía raciones M1.000 a otro establecimiento donde sólo se entregan desayunos. ${ }^{35}$ El ejemplo es aún más extremo si el alumno pasa a un establecimiento (de la misma dependencia) donde el IVE es tan bajos que la cobertura es muy inferior.

En el Gráfico 3, donde se expone el "Efecto Vaso de Leche", se muestra cómo una misma persona puede tener dos curvas de indiferencia dependiendo del IVE con que se le califique en cada región. En el gráfico se observa que la persona alcanza una curva menor, si estando en la región $i$ se mueve a la región $j$. La pérdida del apoyo se produce, ya que el individuo en la región $i$ está matriculado en un establecimiento que tiene un IVE alto y el alumno en cuestión califica para el PAE, en tanto que en la región $j$ llega a matricularse a un establecimiento de la misma dependencia en el cual no está garantizado que reciba PAE, puesto que existe una no uniformidad entre los IVE a nivel regional, provincial y comunal.

\footnotetext{
${ }^{31} \mathrm{La}$ encuesta la responde el profesor a nombre de sus alumnos.

${ }^{32}$ Las variedades de alimentación son (en calorías): 350, 600, 650, 700 y 1.000 .

${ }^{33}$ Información que, por supuesto, es confidencial.

${ }^{34}$ Los alumnos que accederán a los programas de JUNAEB son seleccionados al interior de cada establecimiento educacional por los directivos y/o los profesores. La JUNAEB pone a disposición de ellos orientadores de criterios técnicos y un software computacional para hacer más objetiva la selección.

${ }^{35}$ A partir del año de 2004 existe un mecanismo de asignación más eficiente, ver Apéndice 3.
} 


\section{Modelo Empírico}

El presente modelo destaca la influencia de Subsidios a la Educación en la decisión del migrante. Como se vio, existen distintas categorías de subsidios a la educación. En éstas, la subvención regular es absolutamente irrelevante en un modelo migratorio ${ }^{36} \mathrm{El}$ foco de atención está en aquellos subsidios no portables a priori. Aquí se tienen dos áreas: amenities por la alimentación y amenities por una educación de calidad.

La literatura económica sobre migración encuentra sus fundamentos en el clásico documento de Harris y Todaro (1970), el cual se puede trabajar con un modelo lineal simple. El modelo a desarrollar explota los diferenciales entre las distintas variables interregiones.

Una particularidad en los modelos de migración se da en la definición de conceptos. La migración puede ser neta en cuyo caso es el saldo de inmigrantesemigrantes o puede ser bruta, en cuyo caso es la suma de inmigrantes con emigrantes, es decir, se mide movimiento bruto. Sjaastad (1962) concluye que lo relevante al estimar los retornos de la migración es la migración bruta, no la neta y tampoco las tasas de migración. En este sentido, los modelos de migración neta proporcionan considerablemente menor información que los modelos de migración bruta. ${ }^{37}$

Al momento de llevar a cabo un modelo tipo HT que explota las diferenciales en salarios como gatillador de migración, debe quedar claro que, si $j$ es destino, $i$ es origen, entonces $W_{j}-W_{i}$ indica que, si al aplicar el saldo de esta operación como variable explicativa de migración se obtiene signo positivo, la persona deja la economía de la región $i$ por irse a la economía $j$ donde el salario es mayor. La persona se mueve hacia donde la remuneración de su productividad sea mayor.

Para poder obtener el real efecto de la educación sobre la migración se debe controlar por las otras variables presentes en los modelos clásicos de migración. Aroca y Hewings (2002) utilizan: Tamaño de la población de la región origen y de destino, tasas de desempleo de la región de origen y de destino, proporción de la población urbana, diferencial de salarios y costos de transporte. Para esta última variable lo clásico es usar como proxy la distancia entre regiones, considerando que los costos deberían incrementarse a una tasa decreciente:

$$
C T=\delta_{0}+\delta_{1} \text { Dist }+\alpha_{2} \text { Dist }^{2}
$$

La siguiente parece ser la especificación más $a d$ hoc a la pregunta que se responderá. En este caso, los subsidios a la educación son los de educación primaria y secundaria. Entonces la especificación del modelo:

\footnotetext{
${ }^{36}$ Es un subsidio portable, porque el alumno, esté donde esté, recibe educación gratuita.

${ }^{37} \mathrm{El}$ mismo antecedente es proporcionado en Jackman y Savouri (1992).
} 
(10)

$m_{t, i j}=f\left(P O P_{t, i}, P O P_{t, j}, d_{i, j}, d_{i, j}^{2}, \operatorname{Sal} t_{t, i j}, E s_{t, i j}, \operatorname{Sjun}{ }_{t, i j}, \operatorname{Min}(a)_{t, i j}, \operatorname{Min}(b)_{t, i j} U_{t, i j}, P P U_{t, i}\right)$

El Cuadro 1 muestra la descripción de las variables del modelo.

CUADRO 1

DESCRIPCION DE LAS VARIABLES DEL MODELO

\begin{tabular}{|c|c|c|}
\hline Variables & $\begin{array}{c}\text { Signo } \\
\text { esperado }\end{array}$ & Descripción \\
\hline$m_{t, i j}$ & & $m_{t, i j}=M_{i j} /$ Pop $_{i}$, Con $M_{i j}$ migración entre región $i$ y la región $j$. \\
\hline Pop $_{i}$ & $(-)$ & Población de la región de origen \\
\hline$P_{o p j}$ & $(+)$ & Población de la región de destino \\
\hline$d_{i, j}$ & $(-)$ & Distancia en kilómetros entre regiones \\
\hline$d_{i j}^{2}$ & $(+)$ & Representa la tasa decreciente en el incremento de los costos de emigrar \\
\hline$w_{i j}$ & $(+)$ & Diferencial de salarios entre región de origen $i$ y destino $j . w_{i j}=\left(W_{j}-W_{i}\right)$ \\
\hline$E s_{i j}$ & $(+)$ & Diferencial de nivel de capital humano entre regiones.; $k_{i j}=\left(K_{j}-K_{i}\right)$ \\
\hline $\operatorname{Sjun}_{i j}$ & $(-)$ & Diferencial Subsidio JUNAEB entre regiones; $\operatorname{Sjun}_{i j}=\left(\operatorname{Sjun}_{i}-\right.$ Sjun $\left._{j}\right)$ \\
\hline $\operatorname{Min}(a)_{i j}$ & $(-)$ & Diferencial Subsidio Mineduc "A" entre regiones; $\operatorname{Min}_{i j}=\left(\operatorname{Min}_{i}-\operatorname{Min}_{j}\right)$ \\
\hline $\operatorname{Min}(b)_{i j}$ & $(-)$ & Diferencial Subsidio Mineduc "B" entre regiones; $\operatorname{Min}_{i j}=\left(\operatorname{Min}_{i}-\operatorname{Min}_{j}\right)$ \\
\hline$U_{i j}$ & $(-)$ & Diferencial tasas de desempleo entre regiones. $U_{i j}=\left(U_{j}-U_{i}\right)$ \\
\hline$P P U_{i}$ & $(+)$ & Proporción de población urbana en la región de origen. \\
\hline
\end{tabular}

La columna 2 indica el signo esperado a priori para las variables del modelo. El tamaño de la población se usa para compensar las escalas de medición. Trabajos previos de migración como Barro y Sala-i-Martin (1992b) suelen utilizar la población de origen.

\subsection{Variables del Modelo Empírico, Construcción y Origen de los Datos}

\subsubsection{Migración, población y distancia}

Los datos para la variable dependiente provienen del Censo de Población y Vivienda. El lugar de residencia habitual actual y el que la persona tenía, cinco años antes, es decir, en 1997 (para el Censo 2002), permiten medir la migración tanto interna como internacional. 
En los Censos se detallan, además, los tamaños de población, así como el porcentaje de urbanidad. La información referida a costos de transportes, expresada mediante la distancia entre regiones, indica la distancia en kilómetros entre las capitales regionales y se obtiene del Instituto Geográfico Militar.

\section{Saldos Migratorios ${ }^{38}$}

Por definición, valores positivos de migración neta indican que la población está inmigrando hacia esa región. Valores negativos indican que la población está emigrando de esa región y un valor nulo indica que no hay migración. ${ }^{39}$

Borjas et al. (1992) sugieren que existe un proceso de auto selección según el cual los flujos migratorios no son aleatorios. Las personas calificadas se van hacia aquella región $j$ que demande trabajo calificado y, a la inversa, personas no calificadas que son oriundas de regiones que demandan trabajo calificado deben emigrar hacia una región que demande su tipo de calificación. Este fenómeno -también presente en Chile- sería una posible explicación a la dirección de los saldos migratorios.

En Bhattacharya (2002b) se habla de la sobreurbanización, ${ }^{40}$ la cual genera "desamenities" tales como excesiva concentración de gente, polución, crímenes, delincuencia, etc. Son atributos sociológicos ${ }^{41}$ que, en el largo plazo, entran en la función de utilidad del individuo en un contexto sociológico. La sobreurbanización mal llevada puede provocar que la ciudad sea difícil de administrar y, como consecuencia, se genera migración entre clusters.

\subsubsection{Subsidio JUNAEB}

La variable que se ocupa para indicar el subsidio de la JUNAEB corresponde al PAE. Los datos corresponden a la cobertura por alimentación en cada región, controlando por número de establecimientos municipales y particulares subvencionados de cada región. La información se obtiene de la Junta Nacional de Auxilio Escolar y Becas. ${ }^{42}$

\footnotetext{
${ }^{38}$ Medidos como Migración Neta $=$ Inmigrantes - Emigrantes $($ ver Anexo 2A). Una medición de saldos brutos entrega 156 movimientos, lo que en términos gráficos no es claro de observar. ${ }^{39}$ En estricto rigor indica que la población que emigra de la región es igual a la que inmigra.

40" Over-Urbanization" en Bhattacharya (2002b), presente en Santiago de Chile.

${ }^{41}$ La literatura de migración construida por los sociólogos da fe de esos fenómenos, la línea de la presente investigación es la de la literatura construida por economistas.

${ }^{42}$ Unidad de Gestión de Alimentación, Área de Control de Gestión PAE.
} 
GRAFICO 4

PRODUCTO PER CAPITA (PPC) VS. INDICE DE VULNERABILIDAD (IVE)

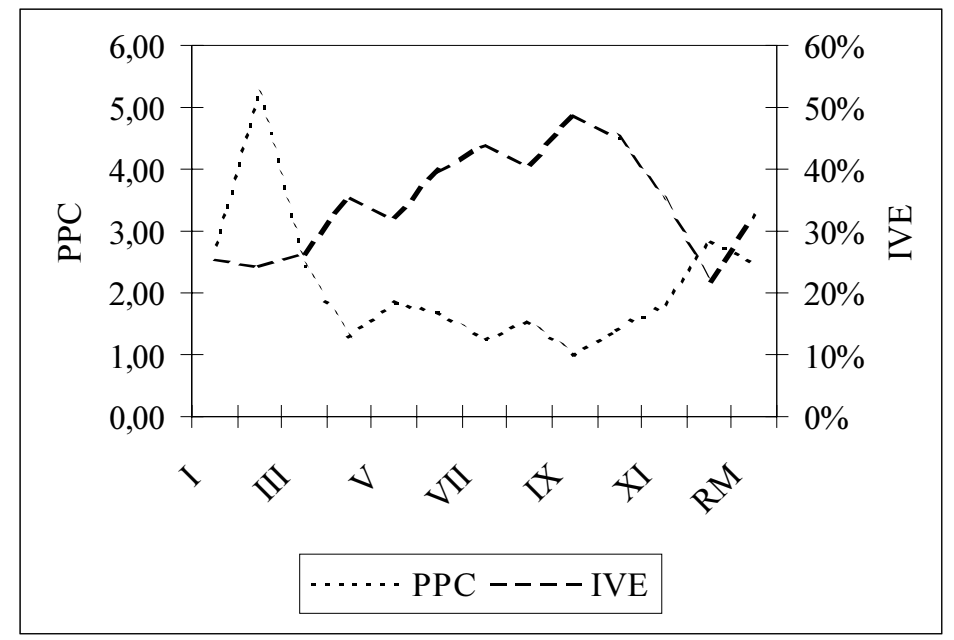

Fuente: PPC a base de BCCh, Marshall et al. (2002) para IVE (Modelo Puntaje).

Al analizar los datos se ve clara la covarianza negativa existente entre el Indice de Vulnerabilidad (IVE) y el Producto per cápita regional. La serie punteada corresponde al PPC Regional, mientras que la serie cortada es la asociada al IVE. Como era de esperar, las regiones más pobres (IX) tienen un IVE más alto, mientras que las regiones ricas tienen un IVE menor. Se espera que un mayor subsidio inhiba la movilidad, por la pérdida de los beneficios en la región de destino, luego el signo esperado es negativo.

CUADRO 2

PORCENTAJE DE ALUMNOS CON NECESIDAD PAE SEGUN EL PROFESOR POR SECTOR (URBANO Y RURAL) Y REGION

\begin{tabular}{crrrcrrr}
\hline \multirow{2}{*}{ Región } & \multicolumn{2}{c}{ Sector } & \multirow{2}{*}{ Total } & \multirow{2}{*}{ Región } & \multicolumn{2}{c}{ Sector } & \multirow{2}{*}{ Rural } \\
& Rural & Urbano & & & & Urbano & \multirow{2}{*}{ Total } \\
\hline I & $75,0 \%$ & $56,1 \%$ & $56,8 \%$ & VIII & $75,8 \%$ & $61,1 \%$ & $61,5 \%$ \\
II & $67,3 \%$ & $40,9 \%$ & $41,1 \%$ & IX & $98,3 \%$ & $78,4 \%$ & $79,1 \%$ \\
III & $100,0 \%$ & $45,2 \%$ & $45,9 \%$ & X & $82,0 \%$ & $67,8 \%$ & $68,9 \%$ \\
IV & $90,4 \%$ & $66,3 \%$ & $66,9 \%$ & XI & $91,5 \%$ & $62,8 \%$ & $64,5 \%$ \\
V & $87,6 \%$ & $58,4 \%$ & $59,3 \%$ & XII & $5,4 \%$ & $48,4 \%$ & $47,4 \%$ \\
VI & $92,8 \%$ & $55,3 \%$ & $56,6 \%$ & RM & $67,1 \%$ & $59,3 \%$ & $59,4 \%$ \\
VII & $83,9 \%$ & $72,2 \%$ & $72,8 \%$ & Total & $81,1 \%$ & $61,1 \%$ & $61,7 \%$ \\
\hline
\end{tabular}

Fuente: Marshall et al. (2002).

El Cuadro 2 muestra la no uniformidad propia al interior de las regiones del país en cuanto a las necesidades de PAE. Los resultados son una consecuencia 
directa de los Indices de vulnerabilidad presenciados en la zona. Se puede observar que la IX Región es donde existe un mayor porcentaje de alumnos con necesidad de PAE.

\subsubsection{Escolaridad}

Como variable proxy del nivel de capital humano regional se utiliza la escolaridad promedio, medida en años de educación. En el cálculo se considera el número de años de escolaridad de la población entre 15 y 64 años, por género, según región.

En el anexo se muestra la heterogeneidad en los años de estudio promedio alcanzado por cada región. Se observa que las regiones con mayor nivel de capital humano son, a su vez, las regiones ricas. Se advierte que las regiones II y XII son las que alcanzan los mayores niveles de educación; mientras que las regiones VII y IX presentan los menores niveles, cercanos a 7,5 años.

Los datos de escolaridad se pueden obtener del Censo Nacional de Población y Vivienda (para una imagen de largo plazo) o de la Encuesta de Caracterización Socioeconómica (CASEN) que realiza el MIDEPLAN. En este caso se optó por la segunda fuente. ${ }^{43}$ Para el cálculo de la escolaridad promedio de la población, el procedimiento se basa, en la confección de una matriz de cálculo en la cual se vacian los datos de la CASEN.

La heterogeneidad de la escolaridad promedio se explica por los Indices de abandono escolar. En este caso, el migrante observa esta señal y decide por el lugar donde la escolaridad sea mayor, es decir, donde las posibilidades de deserción escolar ${ }^{44}$ sean menores. El signo esperado a priori para esta variable es positivo.

\subsubsection{Ingreso}

La variable ingreso es una aproximación del salario regional y en este caso, siguiendo la técnica de Aroca y Hewings (2002), es calculada como la productividad media de la región. Es decir, el PIB regional sobre el número de trabajadores en dicha región, o sea, para el periodo 1997-2002, por ejemplo, se calculan las productividades medias de cada región durante los 5 años y con un promedio simple se obtiene insumo para la regresión. Los datos del PIB regional provienen del Banco Central de Chile, en tanto que los datos de trabajadores son del INE. La variable es incorporada en diferenciales y se esperará un signo positivo.

\footnotetext{
${ }^{43}$ La razón que se esgrime en este caso es la necesidad de datos entre 1992 y 2002.

${ }^{44}$ Ver Apéndice 4.
} 


\subsubsection{Tasas de desempleo}

La variable desempleo es medida con las tasas de desempleo regional, utilizando un promedio simple para los periodos migratorios (1997-2002). El insumo utilizado corresponde a la tasa de desempleo para el trimestre móvil marzo-mayo que es donde entran los datos censales para Chile. Al incorporar este dato al modelo se hace en diferencias. ${ }^{45}$ Se esperará un signo negativo, ya que a mayor tasa de desempleo en la región de destino $j$, hay menores incentivo a abandonar la región de origen $i$. La información proviene del INE.

\subsubsection{Subsidios MINEDUC}

Los subsidios incorporados a la especificación corresponden al Programa de Internado Escolar MIN (A) y al Programa de Subvención por Reforzamiento Educativo MIN (B) ambos a cargo del Ministerio de Educación. En los dos casos, la metodología que se usó para la construcción de las series fue similar a la empleada en el caso del subsidio JUNAEB, es decir, controlando por el universo total. Para el Programa de Internado MIN (A) se toman los datos del periodo, el número de alumnos que reciben subvención de internado y se hace un promedio simple (por ejemplo, entre 1987-1992), para obtener el numerador del input que irá a la serie definitiva. El denominador, que es el que controla escala, es el universo total de matriculados en la región seleccionada. El mismo ejercicio es correcto si se utiliza escuelas que dan Programa de Internado y luego se controla por el número de escuela total de la región. Para el Programa de Subvención por Reforzamiento Educativo MIN (B), se tomó el número de beneficiados (medidos como alumnos) y se controló por universo regional. ${ }^{46} \mathrm{El}$ signo esperado a priori es negativo.

\subsection{Estimación del Modelo}

La metodología de estimación consiste en llevar a cabo regresiones de corte transversal para intervalos liderados por los periodos de tiempo en que se registran los datos migratorios en Chile. El análisis primario en lo referente a interpretación, calidad de la estimación, etc., se realiza para el corte 1997-2002. Se esgrimen dos razones para esto: mayor disponibilidad de información y un concepto de la realidad contemporánea. A su vez, se presenta el corte 1987-1992 para analizar consistencia en el tiempo.

\footnotetext{
${ }^{45}$ Bhattacharya (2002a) también utiliza esta especificación "a la Harris-Todaro".

${ }^{46}$ Para el caso de los subsidios MIN (A) y MIN (B) se tiene el problema de muestra truncada. No se cuenta con la información que se desearía, faltando observaciones, en este caso períodos temporales. En el caso MIN (A) el truncamiento es tal, que se observa el valor exacto para las variables sólo entre 1987-1992 y no se tienen observaciones fuera de ese rango. En el caso MIN (B), el truncamiento es hacia atrás; sólo se observan valores para el rango comprendido entre 1997-2002. Bajo OLS, el truncamiento es generatriz de estimadores sesgados, una matriz covarianza X más grande y $\sigma_{\beta}$ más grande. Greene (1998) sugiere como metodología alternativa y de solución el método de estimación por Máxima Verosimilitud.
} 
CUADRO 3

RESULTADOS

\begin{tabular}{|c|c|c|c|c|c|}
\hline \multicolumn{6}{|c|}{ Migración interregional: regresiones de corte transversal } \\
\hline \multirow[b]{2}{*}{ Variable independiente } & \multicolumn{4}{|c|}{ Variable dependiente: $\ln \left(\mathrm{M}_{\mathrm{ij}} / \mathrm{Pop}_{\mathrm{ii}}\right)$} & \multirow[b]{2}{*}{ Ec5 } \\
\hline & Ec1 & $\mathrm{Ec} 2$ & Ec3 & Ec4 & \\
\hline $\mathrm{C}$ & $\begin{array}{r}-9.05 \\
(-12.40)\end{array}$ & $\begin{array}{r}-9.22 \\
(-12.60)\end{array}$ & $\begin{array}{r}-8.30 \\
(-14.16)\end{array}$ & $\begin{array}{r}-9.43 \\
(-14.89)\end{array}$ & $\begin{array}{r}-10.03 \\
(-12.36)\end{array}$ \\
\hline $\mathrm{POP}_{\mathrm{i}}$ & $\begin{array}{r}-0.01 \\
(-2.51)\end{array}$ & $\begin{array}{r}-0.02 \\
(-2.84)\end{array}$ & $\begin{array}{r}-0.04 \\
(-5.92)\end{array}$ & $\begin{array}{r}-0.01 \\
(-2.68)\end{array}$ & $\begin{array}{r}-0.01 \\
(-2.00)\end{array}$ \\
\hline $\mathrm{POP}_{j}$ & $\begin{array}{r}0.04 \\
(7.23)\end{array}$ & $\begin{array}{r}0.04 \\
(7.45)\end{array}$ & $\begin{array}{r}0.07 \\
(10.73)\end{array}$ & $\begin{array}{r}0.04 \\
(8.40)\end{array}$ & $\begin{array}{r}0.03 \\
(6.44)\end{array}$ \\
\hline DIST & $\begin{array}{r}-0.00 \\
(-4.20)\end{array}$ & $\begin{array}{r}-0.00 \\
(-4.26)\end{array}$ & $\begin{array}{r}-0.00 \\
(-4.56)\end{array}$ & $\begin{array}{r}-0.00 \\
(-5.47)\end{array}$ & $\begin{array}{r}-0.00 \\
(-5.46)\end{array}$ \\
\hline DIST2 & $\begin{array}{r}0.00 \\
(2.03)\end{array}$ & $\begin{array}{r}0.01 \\
(2.04)\end{array}$ & $\begin{array}{r}0.01 \\
(2.14)\end{array}$ & $\begin{array}{r}0.01 \\
(3.28)\end{array}$ & $\begin{array}{r}0.01 \\
(2.89)\end{array}$ \\
\hline W & $\begin{array}{r}7.81 \\
(3.37)\end{array}$ & $\begin{array}{r}7.74 \\
(3.36)\end{array}$ & $\begin{array}{r}5.89 \\
(2.61)\end{array}$ & $\begin{array}{r}5.15 \\
(2.28)\end{array}$ & $\begin{array}{r}6.27 \\
(2.70)\end{array}$ \\
\hline ES & $\begin{array}{r}0.49 \\
(2.12)\end{array}$ & $\begin{array}{r}0.77 \\
(2.72)\end{array}$ & --------- & $\begin{array}{r}0.77 \\
(3.51)\end{array}$ & $\begin{array}{r}0.80 \\
(2.80)\end{array}$ \\
\hline PPU & $\begin{array}{r}4.56 \\
(4.78)\end{array}$ & $\begin{array}{r}4.79 \\
(5.00)\end{array}$ & $\begin{array}{r}4.46 \\
(5.46)\end{array}$ & $\begin{array}{r}4.94 \\
(5.69)\end{array}$ & $\begin{array}{r}5.78 \\
(5.69)\end{array}$ \\
\hline SJUN & $\begin{array}{r}-4.28 \\
(-3.29)\end{array}$ & $\begin{array}{r}-5.37 \\
(-3.72)\end{array}$ & $\begin{array}{r}-2.48 \\
(-2.44)\end{array}$ & $\begin{array}{r}-4.74 \\
(-3.82)\end{array}$ & $\begin{array}{r}-5.23 \\
(-4.01)\end{array}$ \\
\hline MIN (A) & --------- & --------- & $\begin{array}{r}-0.04 \\
(-2.47)\end{array}$ & --------- & -------- \\
\hline MIN (B) & ---------- & $\begin{array}{r}-1.68 \\
(-1.69)\end{array}$ & --------- & ---------- & $\begin{array}{r}-3.92 \\
(-2.97)\end{array}$ \\
\hline UNEMPLOY & $\begin{array}{r}-0.11 \\
(-3.14)\end{array}$ & $\begin{array}{r}-0.07 \\
(-1.55)\end{array}$ & $\begin{array}{r}-0.59 \\
(-4.78)\end{array}$ & $\begin{array}{r}-0.07 \\
(-2.15)\end{array}$ & $\begin{array}{r}-0.09 \\
(-1.94)\end{array}$ \\
\hline R-squared & 0.64 & 0.65 & 0.65 & 0.67 & 0.59 \\
\hline Adjusted R-squared & 0.62 & 0.62 & 0.62 & 0.65 & 0.56 \\
\hline S.E. of regression & 0.82 & 0.82 & 0.84 & 0.799 & 0.89 \\
\hline Sum squared resid & 99.23 & 97.31 & 103.18 & 88.12 & 110.04 \\
\hline Log likelihood & -186.07 & -184.54 & -189.11 & ------ & -------- \\
\hline Durbin-Watson stat & 0.96 & 0.98 & 1.05 & 0.98 & 0.88 \\
\hline Mean dependent var & -5.98 & -5.98 & -5.58 & -6.01 & -6.02 \\
\hline S.D. dependent var & 1.33 & 1.33 & 1.37 & 1.33 & 1.34 \\
\hline J-statistic & -------- & ------ & -------- & 0.05 & 0.07 \\
\hline F-statistic & 28.80 & 26.54 & 29.64 & -------- & -------- \\
\hline Prob(F-statistic) & 0.00 & 0.00 & 0.00 & --------- & --------- \\
\hline
\end{tabular}

Jarque-Vera $=2.87$, valor $\mathrm{p}=0.23$; Test de White $=52.42$, valor $\mathrm{p}=0.49$; Test de Ramsey (fitted terms x 3) $=2.24$, valor $\mathrm{p}=0.086$.

Ec1: 1997-2002 (OLS), Ec2: 1997-2002 (OLS), Ec3: 1987-1992 (OLS), Ec4: 1997-2002 (GMM-Ec1), Ec5: 1997-2002 (GMM-Ec2)

GMM-Ec1: $N^{\circ}$ de Variables x 9; $\mathrm{N}^{\circ}$ de Instrumentos x 14; 5 Restricciones Sobreidentificadas Test " J" de Hansen: $\mathrm{H}_{0}$ : F.O $>0$.

Si $\mathrm{V}_{\mathrm{C}}>\mathrm{V}_{\mathrm{T}}$ Rechazo, el modelo es malo. $\left\{\mathrm{V}_{\mathrm{T}}=0.412\right\}$

GMM-Ec2: $N^{o}$ de variables x 10; $N^{o}$ de Instrumentos x 20; 10 Restricciones Sobreidentificadas, Test "J" de Hansen: $\mathrm{H}_{0}$ : F.O $>0$.

Si $\mathrm{V}_{\mathrm{C}}>$ Vt Rechazo, el modelo es malo. $\left\{\mathrm{V}_{\mathrm{T}}=0.412\right\}$ 
CUADRO 4

MATRIZ DE CORRELACIONES MUESTRALES ${ }^{47}$

\begin{tabular}{|c|c|c|c|c|c|c|c|}
\hline & $\mathrm{POP}_{i}$ & $\mathrm{POP}_{j}$ & W & ES & PPU & SJUN & UNEMPLOY \\
\hline $\mathrm{POP}_{i}$ & 1 & $\begin{array}{r}0.01 \\
(-1.04)\end{array}$ & $\begin{array}{r}0.00 \\
(0.59)\end{array}$ & $\begin{array}{r}0.05 \\
(-2.70)\end{array}$ & $\begin{array}{r}0.09 \\
(3.98)\end{array}$ & $\begin{array}{r}0.00 \\
(-0.69)\end{array}$ & $\begin{array}{r}0.073 \\
(3.48)\end{array}$ \\
\hline $\mathrm{POP}_{j}$ & & 1 & $\begin{array}{r}0.00 \\
(-0.59)\end{array}$ & $\begin{array}{r}0.05 \\
(2.70)\end{array}$ & $\begin{array}{r}0.00 \\
(-0.32)\end{array}$ & $\begin{array}{r}0.00 \\
(0.69)\end{array}$ & $\begin{array}{r}0.07 \\
(-3.48)\end{array}$ \\
\hline W & & & 1 & $\begin{array}{r}0.45 \\
(11.27)\end{array}$ & $\begin{array}{r}0.23 \\
(-6.86)\end{array}$ & $\begin{array}{r}0.57 \\
(14.23)\end{array}$ & $\begin{array}{r}0.00 \\
(0.21)\end{array}$ \\
\hline ES & & & & 1 & $\begin{array}{r}0.17 \\
(-10.52)\end{array}$ & $\begin{array}{r}0.81 \\
(25.54)\end{array}$ & $\begin{array}{r}0.17 \\
(-5.57)\end{array}$ \\
\hline PPU & & & & & 1 & $\begin{array}{r}0.43 \\
(-10.88)\end{array}$ & $\begin{array}{l}0.057 \\
(3.05)\end{array}$ \\
\hline SJUN & & & & & & 1 & $\begin{array}{r}0.02 \\
(-1.74)\end{array}$ \\
\hline UNEMPLOY & & & & & & & 1 \\
\hline
\end{tabular}

\subsubsection{Discusión de los resultados}

En general, se puede decir que los parámetros estimados tienen el signo esperado y son en su totalidad estadísticamente significativos, contribuyendo a que la regresión explique sobre el $60 \%$ de la variación. La variable población tiene el signo esperado respaldando la hipótesis del "Efecto Luces de la Ciudad". La variable distancia y su cuadrado tienen el signo esperado, respaldando la hipótesis del "Efecto Distancia". La variable ingreso tiene el signo esperado y es la que tiene el efecto mayor. La variable Subsidio JUNAEB y la variable MIN $(B)^{48}$ tienen el signo esperado, respaldando la hipótesis del "Efecto Vaso de Leche". La variable escolaridad tiene el signo esperado, respaldando el "Efecto Capital Humano". El coeficiente asociado a la proporción de la población urbana sugiere que las personas que habitan en ciudades más urbanizadas tendrán una mayor probabilidad de migrar. La variable desempleo tiene el signo esperado, respaldando el "Efecto Información".

Sobre la base de la información del Cuadro 4, no se evidencia presencia explícita del problema de multicolinealidad entre las variables explicativas. De hecho, el único par que tiene una correlación sobre el promedio es el Subsidio JUNAEB con escolaridad promedio, el cual estaría dado por la importancia que tiene la ayuda del mencionado subsidio en familias que ven esa ayuda como la única fuente de alimento para los hijos, de manera que a mayor ayuda menor deserción escolar. Debido a que no presenta relación con alguna otra variable, se decide dejarla presente en la especificación, considerando que es significativa, tiene el

${ }^{47}$ Correspondientes a la ecuación 1 .

${ }^{48}$ Resultado en Ec2 y Ec5, no se incluye MIN (A) en la Ec2 y Ec5 por no disponer de información. En la ecuación para 1987-1992 se incorporó MIN (A) y resulta significativo y con el signo esperado. 
signo esperado y, además, al hacer un Test de Variables Irrelevante ${ }^{49}$ no se puede rechazar la hipótesis nula (Variable no pertenece al modelo). Sumado a esto, al hacer el Test de Ramsey Reset con fitted $x 3$ resulta en una correcta especificación. Al llevar a cabo un test de normalidad de los errores vía Test de Jarque Vera, se puede ver que existe evidencia de normalidad en los errores (no es posible rechazar la hipótesis nula). El Test de White para Heterocedasticidad no denota presencia del problema vinculado con los errores de la regresión. Sin embargo, existiría evidencia de un patrón de autocorrelación espacial (datos de corte transversal) positiva. El indicio lo da el valor del estadístico de Durbin y Watson y luego se corrobora con un Test Multiplicador de Lagrange del tipo Breusch-Godfrey con más de 5 rezagos $^{50}$ (considerando la estructura espacial). El resultado no sorprende, pues en trabajos anteriores de convergencia regional evidencian problemas para las regiones de Chile en cuanto a la existencia de autocorrelación espacial; ${ }^{51}$ Higuera (2004) trabaja con los estadísticos "I Moran Globales y Locales" encuentra evidencia de autocorrelación espacial positiva, es decir, las regiones con niveles de PIB per cápita similares tienden a estar unidas. La corrección que yo realizo está en usar la "Matriz de White", aplicar el Método Generalizado de Momentos (GMM), el cual asegura estimaciones eficientes aún en presencia de errores no esféricos, los resultados (Ec4 y Ec5) son similares a los que se obtienen por OLS, sin autocorrelación. Los signos esperados son todos correctos y la significancia estadísticamente aceptada a un tamaño nominal del 5\%.

\section{CONCLUSIONES}

El objetivo de este artículo ha sido encontrar una respuesta a la pérdida de convergencia regional en Chile. La pregunta inicial fue: ¿Cómo una política -en particular la educación-puede inducir o inhibir la migración? El argumento fue que la poca migración interregional sería lo que provoca la falta de convergencia (Barro y Sala-i-Martin, 1991) y, a su vez, esta falta de movilidad sería explicada -en parte- por los Subsidios a la Educación.

El principal resultado de la investigación se generó al introducir la dimensión educativa en un modelo como el de Harris-Todaro ampliado en un contexto familiar. El subsidio alimenticio que otorga (hace ya 40 años) el Ministerio de Educación, a través de la Junta Nacional de Auxilio Escolar y Becas (JUNAEB), es

\footnotetext{
${ }^{49}$ Redundant Variables: Esc $\mathrm{H}_{0}$ : Variable es Irrelevante. F-Statistic: 4.4748, valor $\mathrm{p}=0.036$

${ }^{50}$ Breusch Godfrey Lagrange Multiplier Test (x 5): 12.00 , valor $\mathrm{p}=0.034$

${ }^{51}$ En Aroca y Hewings (2002) no se entrega información sobre la posibilidad del fenómeno de autocorrelación. Trabajos de Morandé et al. (1996) y Fuentes (1996) sí presentan el problema. ${ }^{52}$ El Estadístico "I Moran" es una medida de la asociación entre el PIB per cápita estandarizado y su rezago espacial. Un valor $>0$ en I Moran es indicativo que las regiones de alto (bajo) PIB per cápita tienden a estar situadas junto a regiones de alto (bajo) PIB per cápita. Un valor $<0$ del I Moran refleja lo contrario. Para un análisis más completo se pueden revisar Aroca y Bosch (2000) e Higuera (2004)
} 
un poderoso incentivo para no migrar entre las regiones de Chile. El hecho de que la focalización de la ayuda se construya exclusivamente a base del Indice de Vulnerabilidad (IVE) genera situaciones complejas cuando la familia decide cambiarse de establecimiento, lo cual se acentúa mucho más al cambiarse de región; el temor legítimo-a perder el apoyo del gobierno en materia alimenticia y otros hace que las personas sean poco móviles.

Otros resultados que arrojó la investigación confirman los trabajos de Lewis (1954), Ranis y Fei (1961), Todaro (1969) y Harris y Todaro (1970), enfatizando el rol del Efecto Ingreso al momento de decidir entre regiones. El ajuste en el mercado laboral puede ser visto como una solución laissez faire óptima, al permitir clarear el mercado en muchos casos a través de un proceso de autoselección. La probabilidad de obtener empleo en la región $j$ va depender de las vacantes disponibles en la región $j$ y del número de trabajadores desempleados que sean buscadores de empleo en la región $j$. El proceso de convergencia en producto (o ingresos) per-cápita se verá favorecido si los canales de información son capaces de transmitir de manera oportuna la situación que vive la región $j$ hacia la región $i$.

La metodología que se utilizó para abordar el tema siguió la línea de Aroca y Hewings (2002), confeccionando una regresión para migración que contuviera todos los movimientos migratorios en Chile, medidos como migración bruta.

La estimación de corte transversal obligó a utilizar como variable líder la propia variable dependiente que se recoge como un flujo comprendido en 5 años.

La formación de clubes de convergencia, ya mencionada por Higuera (2004), que le dan una mayor importancia a la geografía propia del país, provocaron la existencia de autocorrelación espacial. El problema en este caso se abordó mediante el uso del Método Generalizado de Momentos (GMM), obteniendo resultados correctos y con pendientes estimadas econométricamente eficientes.

La recomendación de política que se desprende del estudio va en la línea de diseñar subsidios a la educación que sean portables, de modo tal que si el alumno se cambia de región no exista el temor a perder el apoyo. De hecho, el problema generado es mucho más general: la no portabilidad es un incentivo a que los niños no se cambien a establecimientos educacionales mejores, ya que perderían la alimentación escolar en ese sentido el incentivo es perverso, pues no promueve educación de mejor calidad.

Una futura línea de investigación consiste en analizar empíricamente otra clase de subsidios distintos a los de educación. 


\section{REFERENCIAS}

Aroca, P. G. y J. D. Hewings (2002), "Migration and labor market adjustment: Chile, 19771982 and 1987-1992", Annals of Regional Science, 36: 197-218.

Aroca, P. y M. Bosch (2000), "Crecimiento, Convergencia y Espacio en las Regiones Chilenas: 1960-1998", Estudios de Economía, diciembre 27(2): 199-224.

Banco Central de Chile (1998), "Regionalización del Producto Interno Bruto (PIB), Base 1986", Departamento de Cuentas Nacionales.

Barro, R. y X. Sala-i-Martin (1991), "Convergence Across States and Regions", Brookings Papers on Economic Activity, 1:1991, 107-158.

Barro, R. y X. Sala-i-Martin (1992a), "Convergence". The Journal of Political Economy, 100(2): 223-251.

Barro, R. y X. Sala-i-Martin (1992b), "Regional Growth and Migration: A Japan-U.S. Comparison", NBER Working Papers Series, No 4038.

Barro, R y X. Sala-i-Martin (1995), Economic Growth, Mc Graw-Hill Inc, New York.

Becker, G. S. (1993), Human Capital. NBER, Second Edition, New York.

Bernard, A. y S. Durlauf(1995), "Convergence in international Output", Journal of Applied Econometrics 10: 97-108.

Bhattacharya, P. (1993), "Rural-Urban Migration in Economic Development", Journal of Economic Surveys, 7(3): 243-273.

Bhattacharya, P. (1994), "A Multisector Modelo Of LDC", Scottish Journal of Political Economy, 41(3): 225-255.

Bhattacharya, P. (1998), "Migration, Employment and Development: A Three-Sector Analisys", Journal of International Development, 10(7): 899-921.

Bhattacharya, P. (2002a), "Rural-To-Urban Migration in LDCS: A Test of Two Rival Models", Journal of International Development, 14: 951-972.

Bhattacharya, P. (2002b), "Urbanization in Developing Countries", Economic and Political Weekly, October 12, 2002.

Borjas, G. J., S. G. Bronars y S. J. Trejo (1992), "Self-Selection and Internal Migration in The United States", NBER Working Papers Series, No 4002.

Coeymans J. E. (1982), "Determinantes de la Migración Rural-Urbana en Chile Según Origen y Destino", Documentos de Trabajo $N^{o} 81$, Instituto de Economía, Pontificia Universidad Católica de Chile.

Díaz, J. (2002), "Convergencia-Divergencia Regional en Chile: Posibles Causas y Efectos", mimeo, División de Planificación Regional, MIDEPLAN.

Díaz, R. y P. Meller (2003), “Crecimiento Económico Regional en Chile: ¿Convergencia?”, Documentos de Trabajo $N^{\circ} 180$, Centro de Economía Aplicada, Universidad de Chile.

Díaz, R.; A. Pardo y P. Meller (2002), “Análisis Económico-Descriptivo de las Regiones Chilenas", Documentos de Trabajo $N^{o} 133$, Centro de Economía Aplicada, Universidad de Chile.

Fuentes, R. (1996), “Convergen las Regiones en Chile? Una Interpretación”, en F. Morandé y R. Vergara (eds.), Análisis Empírico del Crecimiento en Chile. CEPILADES/Georgetown University.

Gallego, F. (2002), "Competencia y Resultados Educativos: Teoría y Evidencia para Chile", Cuadernos de Economía, 39 (118): 309-352.

Gathak, S.; P. Levine y S.W. Price (1996), "Migration Theories and Evidence An Assesment", Journal of Economics Surveys, 10(2): 159-198.

Gil, A. (2003), "Migración y Expectativas Intergeneracionales", Revista de Análisis Económico, 18 (1): 117-130. 
González, P.; A. Mizala y P. Romaguera (2002), "Recursos Diferenciados a la Educación Subvencionada en Chile", Serie Economía $N^{o} 150$, Centro de Economía Aplicada, Universidad de Chile.

Green, W. (1998), Econometric Analysis, Third Edition, Prentice Hall Inc.

Greenwood, M. J. (1975), "Research on Internal Migration in the United States: A Survey", Journal of Economic Literature, 13(2): 397-433.

Harris, J. R. y M. P. Todaro (1970), "Migration, Unemployment and Development: a TwoSector Analysis", American Economic Review, 60(1): 126-142.

Hicks, J. R. (1948), The Theory of the Wages. Smith, New York.

Higuera, F. (2004), “Análisis del Crecimiento y Convergencia Regional en Chile Empleando un Enfoque Espacial", Tesis de Magíster en Economía Aplicada, Departamento de Ingeniería Industrial, Universidad de Chile.

Jackman, R. y S. Savouri (1991), "Regional Migration in Britain: An Análisis of Gross Flows Using NHS Center Register Data", The Economic Journal, 102: 1433-1450.

Krugman, P. y M. Obstfeld (1994), Internacional Economics: Theory and Policy. $2^{\text {nd }}$ Edition, Mc Graw-Hill Inc, Madrid.

Lewis, W. A. (1954), "Economic Development with Unlimited Supplies of Labour", The Manchester School, May, 22: 139-191.

Marshall, G. y L. Correa (2002), "Modelo de Focalización y Cálculo del Indice de Vulnerabilidad para la Asignación de Raciones del Programa de Alimentación Escolar 2003". Documento elaborado para JUNAEB, Departamento de Salud Pública y Departamento de Estadística, Pontificia Universidad Católica de Chile, noviembre.

Mincer J. (1978), "Family Migration Decisions", Journal of Political Economy, 86 (5): 749-775.

MINEDUC (2004), "Descripción de Pagos Gestionados por la Coordinación Nacional de Subvenciones", Documento Interno Director de Subvenciones.

Mizala, A. y P. Romaguera (2000), "Sistemas de Incentivos en Educación y la Experiencia del SNED en Chile", Documento de Trabajo 82, CEA-U.Chile.

Morandé, F.; R. Soto y P. Pincheira (1996), “Achilles, The Tortoise, and Regional Growth in Chile”, en F.Morandé y R.Vergara (eds.) Análisis Empírico del Crecimiento en Chile, CEP-ILADES/Georgetown University.

Oyarzún, C. y I. Araya (2001), "Long Run Dynamics of Regional Growth in Chile", Estudios de Economía, 28(1): 69-78.

Ranis, G y J.C.H. Fei (1961), “A Theory of Economic Development”, American Economic Review, 51(4): 533-565.

Riffo, L. (1999), "Crecimiento y Disparidades Regionales en Chile: Una visión de Largo Plazo", Estadística y Economía, Instituto Nacional de Estadísticas (INE).

Ritsilä, J. y M. Ovaskainen (2001), "Migration and Regional Centralization of Human Capital", Applied Economics, 33(3): 317-325.

Sapelli, C. (2002), "La Economía de la Educación y el Sistema Educativo Chileno", Cuadernos de Economía, 39(118): 281-296.

Sapelli, C. y C. Aedo (2001), "El Sistema de Vouchers en la Educación, Una Revisión de la Teoría y Evidencia Empírica para Chile", Estudios Públicos, 82.

Sapelli, C. y B. Vial (2002), "The Performance of Private and Public Schools in the Chilean Voucher System", Cuadernos de Economía, 39(118): 423-454.

Sjaastad, L. A. (1962), "The Costs and Returns of Human Migration", Journal of Political Economy, 70(5), Part 2: 80-93.

Todaro, M. P. (1969), “A Model of Labour Migration and Urban Unemployment in Less Development Countries", American Economic Review, 59(1): 138-148. 


\section{APENDICE 1 \\ CONVERGENCIA REGIONAL, ENFOQUEDE SERIES DE TIEMPO}

Un enfoque alternativo para probar convergencia es el Enfoque Econométrico de Series de Tiempo propuesto por Bernard y Darlauf $(1995)^{53}$ quienes estudian las propiedades de las series de producto per cápita en las distintas economías. Según la definición de los autores, los países (o regiones) $i$ y $j$ convergen, si el pronóstico de largo plazo del producto de ambos países es el mismo en el momento $t$, formalmente:

$$
\lim _{k \rightarrow \infty} E\left(\left(y_{i, t+k}-y_{j, t+k}\right) / I_{t}\right)=0
$$

donde $y_{i}$ corresponde al logaritmo natural del producto per cápita de la economía $i$, con $I_{t}$ representante de la estructura de información para calcular el valor esperado. De acuerdo a esta definición, si dos series de producto per cápita tienen raíz unitaria, sólo convergerían si están cointegradas con vector de cointegración $(1,-1)$.

\section{APENDICE2 \\ MIGRACIONY CONVERGENCIA REGIONAL}

La relación entre migración y convergencia regional ha sido empíricamente probada tanto a nivel internacional como para el caso de Chile. El fundamento apunta a que la migración entre regiones generaría una asignación eficiente de los recursos con lo cual se potencia el crecimiento de la región.

La técnica que se suele usar para obtener el impacto sobre las fórmulas neoclásicas consiste en especificar empíricamente la fórmula (1), la cual al reordenar queda:

(b) $\quad \frac{\ln \left(y_{i T}\right)-\ln \left(y_{i 0}\right)}{T}=\alpha-\frac{1-e^{\beta T}}{T} \ln \left(y_{i 0}\right)+\mu_{i 0, T}$

Donde el lado izquierdo representa la tasa de crecimiento promedio del producto (o ingreso) per cápita ${ }^{54}(y)$ para la región i, en un intervalo entre 0 y T. En (12) se tiene que:

\footnotetext{
${ }^{53}$ Bernard y Durlauf (1995).

${ }^{54}$ La relación inversa entre la tasa de crecimiento y el nivel de producto (o ingreso) per cápita inicial es una implicación directa de la existencia de retornos decrecientes en la acumulación de capital.
} 
(c) $\quad a=x+\left[\frac{\left(1-e^{-\beta T}\right)}{T} \ln \left(y^{S S}\right)\right]$

Donde, $y^{S S}$ representa el valor del producto per cápita en estado estacionario y $x$ corresponde a la tasa de crecimiento en estado estacionario.

Para el caso chileno, Fuentes (1996) incorpora la migración en la ecuación (b), mediante una nueva variable en el lado derecho. Dado que el análisis de convergencia obliga a usar 13 observaciones por año, en su análisis está presente el problema de muestra pequeña. En efecto, al incorporar migración neta ${ }^{55}$ y ocupar 13 datos por periodo de tiempo se necesita utilizar técnicas de panel para abarcar un periodo estadísticamente significativo (recordando siempre que la migración se mide en los Censos). Fuentes ocupa información desde 1960 hasta 1990 y estima mediante Mínimos Cuadrados No Lineales.

Para el caso internacional, los trabajos de Barro y Sala-i-Martin (1991) y (1992b) usan, en rigor, la misma técnica.

\section{APENDICE 3 \\ SISTEMANACIONALDEASIGNACIÓN CON EQUIDAD (SINAE) ${ }^{56}$}

El SINAE es un programa que va dirigido a transparentar el proceso de asignación de apoyo, permitiendo evaluar de forma individualizada la condición de desventaja de cada estudiante. Hasta antes del SINAE el método de focalización se basaba exclusivamente en el IVE; el SINAE se complementa con el IVE y facilita técnicamente la labor del educador que debe decidir quién recibe alimentos y quién no. Con el SINAE se entrega un listado de los estudiantes que deben recibir las raciones de acuerdo a su prioridad de atención. El método permite evaluar el impacto de los distintos programas de la JUNAEB; el escolar que recibe la beca está claramente identificado y puede continuar con el beneficio mientras lo requiera, incluso si cambia de escuela o lugar de residencia.

\footnotetext{
${ }^{55}$ También se podría ocupar migración bruta y realizar una metodología similar a la expuesta en el desarrollo de este artículo con un vector de 156 observaciones y luego estimar por mínimos cuadrados no lineales.

${ }^{56}$ Programa que se implementó a comienzos del año 2004.
} 


\section{APENDICE 4}

A. Saldos Migratorios (Netos) 1997-2002

(Miles de personas)

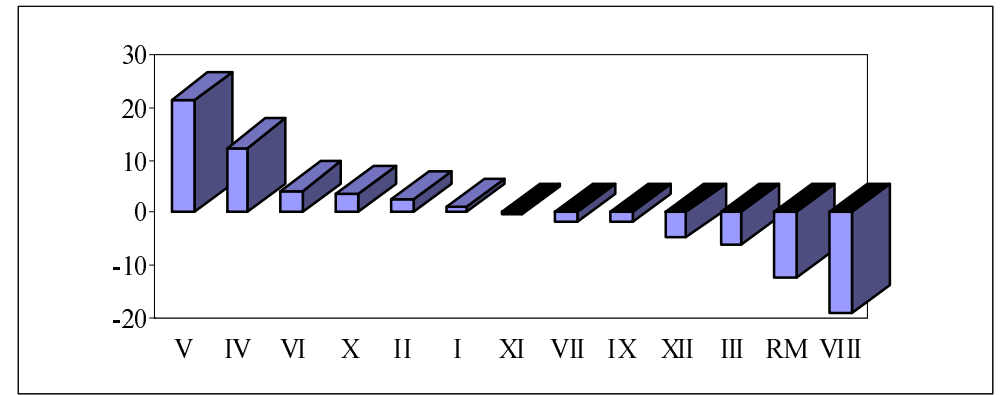

Fuente: Elaboración Propia a base del Censo 2002, INE.

B. Escolaridad Promedio (años), Tasas de Aprobación, Reprobación y Abandono Escolar

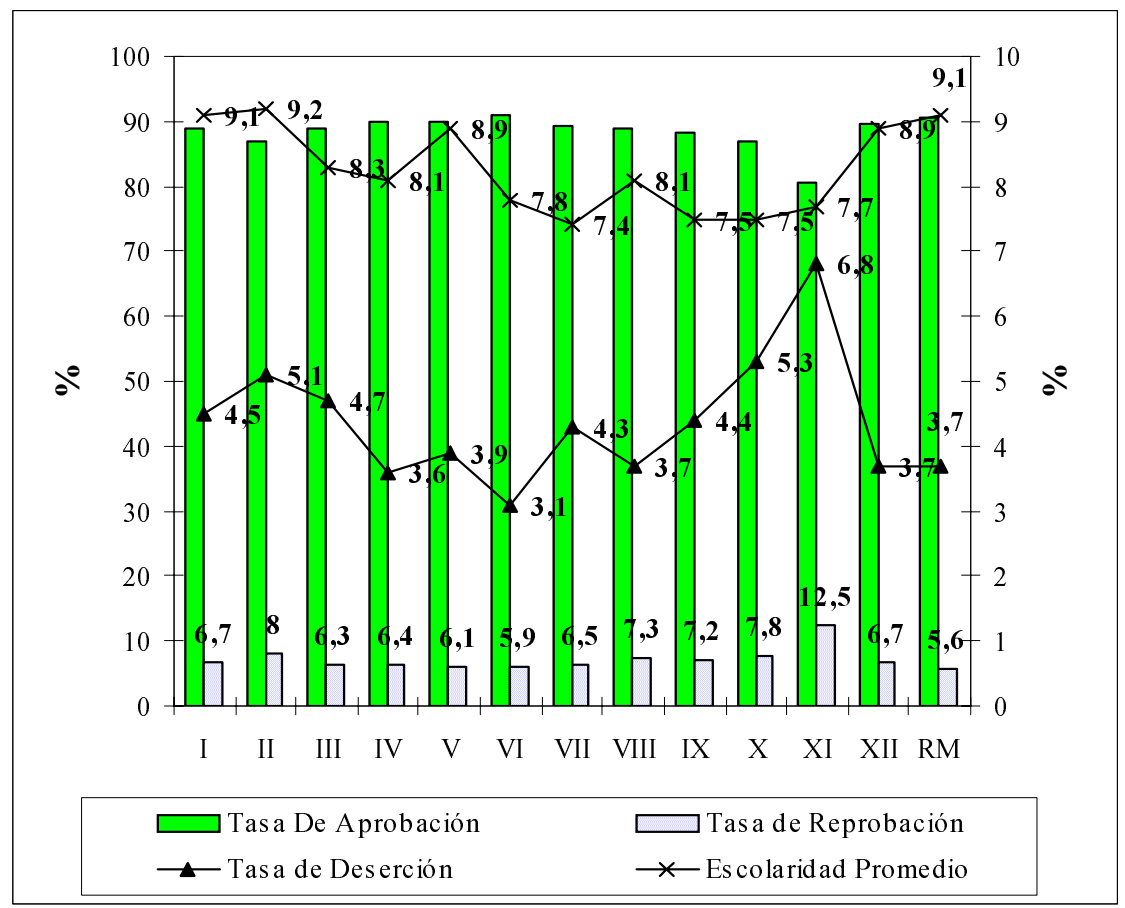

Tasa de Aprobación, Reprobación y Abandono por nivel de enseñanza (media total). Fuente: Mineduc, Indicadores de la Educación en Chile (2002), Censo (2002) para Escolaridad Promedio. 